\section{(1) \\ CrossMark}

\title{
To progress understanding of disease triggers and modifiers in sarcoidosis, stratification is the key
}

\author{
Coline H.M. van Moorsel
}

Affiliation: Interstitial Lung Diseases Center of Excellence, Dept of Pulmonology, St. Antonius Hospital, Nieuwegein, The Netherlands.

Correspondence: Coline H.M. van Moorsel, Interstitial Lung Diseases Center of Excellence, Dept of Pulmonology, St. Antonius Hospital, Koekoekslaan 1, Nieuwegein 3435CM, The Netherlands.

E-mail: c.van.moorseldantoniusziekenhuis.nl

@ERSpublications

To progress understanding in sarcoidosis, stratification is the key: a call for disease phenotyping http://ow.ly/k0T630gCgOb

Cite this article as: van Moorsel CHM. To progress understanding of disease triggers and modifiers in sarcoidosis, stratification is the key. Eur Respir J 2017; 50: 1702002 [https://doi.org/10.1183/ 13993003.02002-2017].

The vast majority of sarcoidosis literature starts with something similar to the following sentence "Sarcoidosis is a multi-system granulomatous disease of unknown aetiology predominantly affecting the lung". The unknown cause continues to inspire researchers to search for a possible trigger, especially if new and high-throughput methods are at their disposal. The histological hallmark of sarcoidosis is epithelioid cell granuloma. Formation of granuloma is a common reaction towards, for example, bacterial agents. Evidence for bacterial involvement in sarcoidosis consist of presence of bacterial peptides inside lung granuloma and other products derived from sarcoidosis patients, the ability of these products to produce a granulomatous reaction in human and in mice, and the success of anti-microbial therapy in patients $[1,2]$. Although active infection has not been found, bacteria, and in particular mycobacteria, are thought to be a causative or disease modifying factor in sarcoidosis.

High-throughput molecular methods for identification of the complete bacterial microbiome in bronchoalveolar lavage (BAL) have recently been applied to sarcoidosis, but have not identified a clear disease-associated dysbiotic profile. GARZONI et al. [3] studied the microbiome in interstitial lung disease, and did not find disordered airway microbiota in sarcoidosis $(n=7)$. A report by ScHeR et al. [4] described the microbiome in immune-mediated disease and concluded that distal airway dysbiosis in untreated early rheumatoid arthrithis was similar to that seen in sarcoidosis $(\mathrm{n}=10)$. Recently a study by CLARKE et al. [5] analysed the microbiome content in lymph node biopsies, but also 16 BAL samples from sarcoidosis subjects and did not identify significantly different bacterial communities between BAL samples from sarcoidosis and controls subjects.

In this issue of the European Respiratory Journal, Zimmermann et al. [6] present the largest study so far and describe the microbial composition in 71 patients with sarcoidosis, 10 healthy controls and 15 IPF patients. Mycobacterial DNA was only present in two sarcoidosis patients. Two taxa were more frequent in

Received: Sept 302017 | Accepted after revision: Nov 032017

Support statement: Funded by ZonMW-TopZorg St Antonius Care grant 842002003. Funding information for this article has been deposited with the Crossref Funder Registry.

Conflict of interest: None declared.

Copyright CERS 2017 
sarcoidosis than in controls: Atopobium sp. and Fusobacterium sp., both potentially alive and active. However, interpretation of these results is difficult because both taxa have also been described to associate with other lung diseases, such as chronic obstructive pulmonary disease [7] and cystic fibrosis [8].

While researchers are just beginning to understand what constitutes a healthy lung microbiome, they simultaneously try to interpret findings in disease-associated microbiomes. Fusobacterium, for instance, is by some regarded as a true pathogen that should always be treated, while others describe it to be part of a healthy microbiome [9].

The lung microbiome largely overlaps with the oral microbiome, and is the result of microbial immigration, elimination and relative reproduction rates $[10,11]$. Microbiome communities are complex and dynamic and vary throughout life under the influence of the season, geography, local environment, body site and host characteristics like genotype, ethnicity, age, sex, body mass index, diet, drugs and health status [9-12]. This wide variety of influences makes it difficult to develop studies that account for all these factors. It is therefore surprising that microbiome studies continuously keep comparing their findings in disease with those in health.

In inflammatory bowel disease, LEVI et al. [13] call for a more individualised approach that links microbial community structure and disease phenotypes. Comparison between phenotypes might be more valuable than differentiation between patients and healthy subjects. The value of the microbiome may lie in differentiating subtypes that share microbiome signatures indicative for prognosis or treatment. Such an approach requires stratification within patient cohorts.

Because the sample size in the Zimmermann et al. [6] study was large, the authors stratified the patients by lung phenotype according to radiographic Scadding stages. In comparison with controls, ZimmERMANN et al. [6] found that the increase in Atopobium sp. was associated with stage I $(n=17)$ and II $(n=39)$ and the increase in Fusobacterium sp. with stage II and III $(n=6)$ of sarcoidosis. The observation that disease-associated pathogens may differ per stage is interesting. But, whether Atopobium is truly associated with hilar lymphadenopathy and Fusobacterium with parenchymal involvement in sarcoidosis requires further investigation, ideally in a study designed to sufficiently power strata for direct comparison amongst each other instead of with controls.

The value of stratification in sarcoidosis is well illustrated by looking at what, biologically, can be called specific phenotypes of sarcoidosis: Blau syndrome, chronic beryllium disease and Löfgren syndrome.

Blau syndrome is an inflammatory disorder characterised by the triad of granulomatous arthritis, uveitis and dermatitis [14]. It is a familial monogenic disease with autosomal dominant inheritance pattern thought to be caused by gain of function mutations in NOD2 that result in the continuous stimulation of nuclear factor $\mathrm{\kappa B}[15]$.

Chronic beryllium disease is a granulomatous lung disease caused by exposition to beryllium. The disease is clinically indistinguishable from pulmonary sarcoidosis and nowadays extrapulmonary manifestations are regarded rare [16]. Carriership of human leukocyte antigen (HLA)-DPB1 E69 contributes significantly to the risk of disease with odds ratios for E69 homozygotes versus non-carriers of over 20 [17].

Löfgren syndrome is an acute form of sarcoidosis characterised by erythema nodosum, bilateral hilar lymphadenopathy (BHL), and bilateral ankle arthritis or periarticular inflammation. This syndrome is common among Caucasians and has a good prognosis [18]. The trigger is unknown but disease occurrence is seasonal and disease susceptibility and prognosis are highly dependent on carriership of HLA-DRB ${ }^{\star} 0301$ genotype [19].

There are three commonalities between Blau syndrome, chronic beryllium disease and Löfgren syndrome: first, the necessity to cluster patients prior to identification of factors causal to disease; secondly, organ involvement is informative for the identification of disease phenotypes, particularly in combination with demographics and disease course; and thirdly, genetic association with each specific phenotype is very high.

Genetic penetrance, meaning what percentage of patients with the variant get the disease, is extremely high for the NOD2 mutations, but extremely low for the HLA-types associated with chronic beryllium disease and Löfgren syndrome. Low penetrance is a sign that, apart from the HLA-type, other factors, genetic or environmental, play a key role in development of disease. In chronic beryllium disease, the environmental agent was identified by occupational clustering, for others forms of sarcoidosis bacteria might be such an environmental key factor.

BTNL2 is a disease risk locus for sarcoidosis [20]. In the study by ZimmermanN et al. [6], stratification of patients by genotype of BTNL2 (rs2076530) showed that carriership of the risk allele A was significantly associated with a decrease in bacterial burden. BTNL2 is part of the HLA region and has 
immunomodulating properties. BTNL2 variants have been associated with many immune mediated diseases, e.g. ulcerative colitis, Graves' disease, type 1 diabetes, asthma, systemic lupus erythematosus, and rheumatoid arthritis, whether or not secondary to HLA-DRB1 alleles [21].

Odds ratios for BTNL2 in sarcoidosis however, are low, much lower than many risks associated with HLA types in sarcoidosis phenotypes [22]. HLA class II molecules, like DPB1 and DRB1, are involved in the presentation of peptides derived from extracellular proteins. In search for combinations between disease triggers and host genetics, analysis of HLA class II variation would be a first choice. The ACCESS study combined detailed environmental histories with high resolution HLA class II typing and found that HLA DRB1 ${ }^{\star} 1101$ and insecticide exposure at work was associated with extrapulmonary sarcoidosis, specifically cardiac sarcoidosis and hypercalcaemia and that HLA DRB1 ${ }^{\star} 1101$ and exposure to molds and musty odours was associated with pulmonary only sarcoidosis [23]. This is the result of a large, laborious and well-designed study and one of the rare examples where clustering (by exposure), localisation (pulmonary versus extra-pulmonary) and genetics (HLA DRB1*1101 positive) come together. Still, translation of the results to the clinic remains difficult.

However, there is something more we can learn from Blau syndrome, chronic beryllium disease and Löfgren syndrome. Genetic expressivity, meaning the extent to which a genetic variant is expressed at the phenotypic level, is relatively uniform. Patients with Blau syndrome, chronic beryllium disease or Löfgren syndrome have clinically clearly defined forms of the disease. There is reason to assume that more, yet to be discovered, clearly defined forms of sarcoidosis may exist.

Defining a phenotypic uniform cohort within this heterogeneous pool of sarcoidosis patients might significantly aid research, even, or perhaps especially, when proportionally small groups of patients can be identified that would easily have been overlooked or that were underpowered in previously performed all-inclusive sarcoidosis studies. Authors suggesting sarcoidosis phenotyping systems have been trying to be as inclusive as possible (reviewed by PEREIRA et al. [24]), but from a scientific perspective the identification of a small, clinically homogeneous group of patients has a better chance of producing clinically applicable results in the future.

In pulmonary fibrosis, a recent research statement formulated criteria for the provisional category "interstitial pneumonia with autoimmune features" [25]. This sparked research on the subject which led, in a very short period of time, to better understanding of the natural history of the disease entity. Currently, there is an unmet scientific need for defining phenotypic clusters of sarcoidosis patients. Therefore, a call for a European Respiratory Society scientific task force to identify one or several phenotypes of sarcoidosis. Successful phenotyping could be key to clinically meaningful and translational results in sarcoidosis.

\section{References}

1 Beijer E, Veltkamp M, Meek B, et al. Etiology and immunopathogenesis of sarcoidosis: novel insights. Semin Respir Crit Care Med 2017; 38: 404-416.

2 Drake WP, Richmond BW, Oswald-Richter K, et al. Effects of broad-spectrum antimycobacterial therapy on chronic pulmonary sarcoidosis. Sarcoidosis Vasc Diffuse Lung Dis 2013; 30: 201-211.

3 Garzoni C, Brugger SD, Qi W, et al. Microbial communities in the respiratory tract of patients with interstitial lung disease. Thorax 2013; 68: 1150-1156.

4 Scher JU, Joshua V, Artacho A, et al. The lung microbiota in early rheumatoid arthritis and autoimmunity. Microbiome 2016; 4: 60.

5 Clarke EL, Lauder AP, Hofstaedter CE, et al. Microbial lineages in sarcoidosis: a metagenomic analysis tailored for low microbial content samples. Am J Respir Crit Care Med 2017; in press [https://doi.org/10.1164/rccm. 201705-0891OC].

6 Zimmermann A, Knecht H, Häsler R, et al. Atopobium and Fusobacterium as novel candidates for sarcoidosis-associated microbiota. Eur Respir J 2017; 50: 1600746.

7 Pragman AA, Kim HB, Reilly CS, et al. The lung microbiome in moderate and severe chronic obstructive pulmonary disease. PLoS One 2012; 7: e47305.

8 Surette MG. The cystic fibrosis lung microbiome. Ann Am Thorac Soc 2014; 11: Suppl. 1, S61-S65.

9 Ubags ND, Marsland BJ. Mechanistic insight into the function of the microbiome in lung diseases. Eur Respir J 2017; 50: 1602467.

10 Dickson RP, Martinez FJ, Huffnagle GB. The role of the microbiome in exacerbations of chronic lung diseases. Lancet 2014; 384: 691-702.

11 Faner R, Sibila O, Agustí A, et al. The microbiome in respiratory medicine: current challenges and future perspectives. Eur Respir J 2017; 49: 1602086.

12 Igartua C, Davenport ER, Gilad Y, et al. Host genetic variation in mucosal immunity pathways influences the upper airway microbiome. Microbiome 2017; 5: 16.

13 Levi M, Kolodziejczyk AA, Thaiss CA, et al. Dysbiosis and the immune system. Nat Rev Immunol 2017; 17: 219-232.

14 Wouters $\mathrm{CH}$, Maes A, Foley KP, et al. Blau Syndrome, the prototypic auto-inflammatory granulomatous disease. Pediatr Rheumatol Online J 2014; 12: 33. 
15 Manthiram K, Zhou Q, Aksentijevich I, et al. The monogenic autoinflammatory diseases define new pathways in human innate immunity and inflammation. Nat Immunol 2017; 18: 832-842.

16 Mayer AS, Hamzeh N, Maier LA. Sarcoidosis and chronic beryllium disease: similarities and differences. Semin Respir Crit Care Med 2014; 35: 316-329.

17 Van Dyke MV, Martyny JW, Mroz MM, et al. Risk of chronic beryllium disease by HLA-DPB1 E69 genotype and beryllium exposure in nuclear workers. Am J Respir Crit Care Med 2011; 183: 1680-1688.

18 Karakaya B, Kaiser Y, van Moorsel CHM, et al. Löfgren's syndrome: diagnosis, management, and disease pathogenesis. Semin Respir Crit Care Med 2017; 38: 463-476.

19 Grunewald J, Brynedal B, Darlington P, et al. Different HLA-DRB1 allele distributions in distinct clinical subgroups of sarcoidosis patients. Respir Res 2010; 11: 25.

20 Valentonyte R, Hampe J, Huse K, et al. Sarcoidosis is associated with a truncating splice site mutation in BTNL2. Nat Genet 2005; 37: 357-364.

21 Arnett HA, Viney JL. Immune modulation by butyrophilins. Nat Rev Immunol 2014; 14: 559-569.

22 Sato H, Woodhead FA, Ahmad T, et al. Sarcoidosis HLA class II genotyping distinguishes differences of clinical phenotype across ethnic groups. Hum Mol Genet 2010; 19: 4100-4111.

23 Rossman MD, Thompson B, Frederick M, et al. HLA and environmental interactions in sarcoidosis. Sarcoidosis Vasc Diffuse Lung Dis 2008; 25: 125-132.

24 Pereira CA, Dornfeld MC, Baughman R, et al. Clinical phenotypes in sarcoidosis. Curr Opin Pulm Med 2014; 20: 496-502.

25 Fischer A, Antoniou KM, Brown KK, et al. An official European Respiratory Society/American Thoracic Society research statement: interstitial pneumonia with autoimmune features. Eur Respir J 2015; 46: 976-987. 